\title{
Probe into Color Preference in Inpatients with Depressive Disorders
}

\author{
Manli Ran ${ }^{1}$, Xiaolin Tan ${ }^{1 *}$, Guoqing Jiang ${ }^{1}$, Xue Chen ${ }^{1}$, Hui Yang ${ }^{1}$, Gang \\ Yuan $^{1}$ \\ ${ }^{1}$ Psychiatry Department of Chongqing Mental Health Center, Chongqing \\ 401147, China.
}

*Corresponding to: Xiaolin Tan. Email: tanxiaolincq@sina.com.

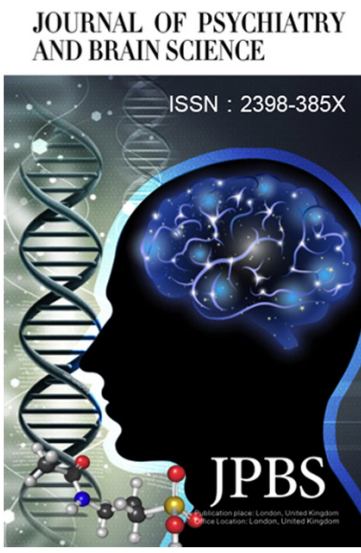

http://jpbs.qingres.com

\section{GOPEN ACCESS}

DOI: $10.20900 / j p b s .20170010$

Received: March 18, 2017

Accepted: May 20, 2017

Published: June 25, 2017

Copyright: @2017 Cain et al. This is an open access article distributed under the terms of the Creative Commons Attribution License, which permits unrestricted use, distribution, and reproduction in any medium, provided the original author and source are credited.

\begin{abstract}
Objectives: To explore the preference of color (abstract and environmental colors) by patients with depression before and after the therapy and the relations between their preferences and depression levels.

Methods: The preference for abstract colors and indoor environment color of 195 depression inpatients and 1005 healthy volunteers was successively tested. Before treatment, HAMD/HAMA was used to assess the level of depression and anxiety. With depression patients, and after six weeks of treatment, the same test tools were used to measure the preference of color and the level of depression and anxiety.
\end{abstract}

Results: The most preference of depression patients for abstract color was blue, the most preference of control groups for abstract color was also blue. Their preference for purple was significantly higher than that of the control group $(p=0.023)$, and their preference for grey was significantly lower than that of the control group $(p<0.001)$. The preference sequence before and after therapy were consistent $(p<0.01)$. The difference of color preference of the patients before and after the therapy was not statistically significant $(p>0.05)$. The most preference of depression patients for indoor environment color was blue, and their preference for purple was higher than that of the control group $(p=0.014)$, and their preference for orange was lower than that of the control group $(p=0.005)$. The preference sequence were consistent before and after therapy $(p<0.01)$, the preference for red and grey decreased after the therapy $(p=0.003,0.040)$ and the preference for yellow increased after the therapy $(p=0.02)$.

Conclusion: The depression patients preferred the cold colors (The most preference of depression patients for color was blue. Their preference for purple was significantly higher than that of the control group. The preference for red and grey decreased after therapy, and the preference for yellow increased after the therapy. A significantly negative correlation was found between yellow preference sequence and HAMD score and between yellow preference sequence and HAMA 
score. A significantly positive correlation was found between red preference sequence and HAMD score , and a significantly negative correlation was found between blue / white preference sequence and HAMD score). These preferences are related to patients' depression levels.

Keywords: Depressive Disorder; Color Preference; Abstract Color; Indoor Environment Color

Colors are everywhere in our daily lives, they delivers psychological stimulus through individual aesthetics. Color is often associated with emotions, such as "depression and blue", "red and anger", etc. Some studies show that the subjects whose Beck Depression Score is greater than 10 tend to choose black or brown to represent their emotions, and subjects who have a higher anxiety score prefer soft colors to relieve their anxiety ${ }^{[1]}$. A survey of Carruthers, et al. ${ }^{[2]}$ shows that people with anxiety and depression will choose grey to represent their sad mood. According to von Goethe ${ }^{[3]}$, a German litterateur, different color tones, warm or cool, can trigger different emotions: the warm ones can inspire liveliness and optimism, while the cool ones can stimulate anxiety and uneasiness. According to research, though patients largely share the same associations of the symbolism of each color, they also have the distinct features of their own that are tightly connected to their physical perception of colors and varied personal experiences. And then, Cognitive factors, especially cognitive bias (Excessive focus on negative information) is one of important factors of the production, persistence and development of the depressive disorder ${ }^{[4]}$. By far, many research shows that there is a connection between the depressed, anxious population and their preference of colors. However, there is no document specifically shows what colors preferred by patients with depression in different emotional states and how they relate to their emotions. Past research on color was only confined to abstract or environmental colors with very limited attentions on depression patients, and very rarely were there any investigative analysis of the two color phenomena. This research aims to discuss the preference of color (abstract and environmental colors) by patients with depression in different emotional states before and after the therapy and the relations between their preferences and depression levels, emotions. The study results are reported below.

\section{SUBJECTS AND METHODS}

\subsection{Subjects}

195 depression inpatients including 94 male and 101 female were selected from Chongqing Mental Health Center from January 2015 to December 2015. They were identified to comply with the major depression diagnosis criteria of "Mental and Behavioral Disorders of International Classification of Diseases (ICD-10) ". They were all Han Chinese and aged 18 - 60 years old, with a mean age of 42.27 \pm 11.70 years old and average education level of $11.66 \pm 3.66$ years. Exclusion criteria: a. patients with color blindness and color amblyopia; b. patients whose organic mental diseases and acute mental disorders cannot be thoroughly investigated. This research experiment was approved by the local ethics committee. The written informed consents were signed by all subjects before the experiment. The difference of gender, age and education level and other data between the two groups was not statistically significant $(p>0.05)$.

Healthy volunteers were recruited through the community during the same period and were identified to be free from mental disorders in accordance with the requirements of ICD-10. The distribution of this control group statistically matched the gender, age and culture of the depression group. There were 1005 health volunteers, including 530 male volunteers and 475 female volunteers, with a mean age of $37.97 \pm 10.47$ years old and average education level of $11.78 \pm 3.74$ years. The informed consents were signed and they were willing to cooperate with the physical examinations see Table1.

Table 1. General Data of Subject Groups

Demographic Characteristics

Subjects Classification

Major Depression $\quad$ Control Group
Group

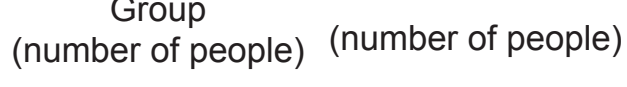

$x^{2}$

$\mathrm{P}$






\begin{tabular}{|c|c|c|c|c|c|}
\hline \multirow[t]{2}{*}{ Gender } & Male & 94 & 530 & 1.343 & 0.246 \\
\hline & Female & 101 & 475 & & \\
\hline \multirow[t]{3}{*}{ Age } & $18-30$ & 43 & 298 & 5.672 & 0.059 \\
\hline & $31-45$ & 87 & 435 & & \\
\hline & $46-60$ & 65 & 272 & & \\
\hline \multirow[t]{3}{*}{ Education Level } & Primary School or Below & 22 & 133 & 2.820 & 0.244 \\
\hline & Secondary School & 110 & 501 & & \\
\hline & University or Above & 63 & 371 & & \\
\hline \multirow[t]{2}{*}{ Marriage } & Married & 147 & 808 & 2.526 & 0.112 \\
\hline & Unmarried & 48 & 197 & & \\
\hline \multirow[t]{2}{*}{ Professional } & Mental & 58 & 350 & 1.880 & 0.170 \\
\hline & Physical Strength & 137 & 655 & & \\
\hline \multirow[t]{2}{*}{ Residence } & City & 49 & 298 & 1.626 & 0.202 \\
\hline & Village & 146 & 707 & & \\
\hline \multirow[t]{2}{*}{ Somatopathy } & Have & 47 & 200 & 1.764 & 0.184 \\
\hline & Not Have & 148 & 805 & & \\
\hline \multirow{3}{*}{$\begin{array}{l}\text { Monthly personal } \\
\text { income }\end{array}$} & $<1000(\mathrm{RMB})$ & 23 & 67 & 10.119 & 0.006 \\
\hline & 1000-2999(RMB) & 149 & 751 & & \\
\hline & $\geq 3000(\mathrm{RMB})$ & 23 & 187 & & \\
\hline
\end{tabular}

195 depression inpatients Clinical Characteristics

\begin{tabular}{|c|c|c|c|c|c|}
\hline \multicolumn{4}{|l|}{ Subjects } & \multirow{2}{*}{$\frac{\mathrm{t}}{\mathrm{l}}$} & \multirow{2}{*}{$\frac{P}{l}$} \\
\hline Classification & First Episode Depression & 88 & l & & \\
\hline & Relapse & 107 & l & & \\
\hline First Episode Age & $12-60$ & $37.45 \pm 11.29$ & l & l & l \\
\hline $\begin{array}{l}\text { Course Of Disease } \\
\text { (Months) }\end{array}$ & $1-362$ & $49.49 \pm 75.38$ & l & l & l \\
\hline \multirow[t]{2}{*}{ HAMD score } & Before Therapy & $25.42 \pm 8.28$ & l & 23.272 & $0.001^{*}$ \\
\hline & After Therapy & $10.18 \pm 9.19$ & l & & \\
\hline \multirow[t]{2}{*}{ HAMA score } & Before Therapy & $16.95 \pm 7.38$ & l & 18.539 & $0.00^{*}$ \\
\hline & After Therapy & $6.68 \pm 6.55$ & I & & \\
\hline
\end{tabular}

Notes: Scale scores (HAMD and HAMA) before and after therapy : * $p<0.01$ " $"$ ': There is no data. 


\subsection{Methods}

\subsubsection{Research Process}

The common questionnaires were self-made to survey the gender, age, marriage, education level and other demographic characteristics of the subjects. Then, professionally trained psychiatrists were invited to test the subjects' preference for abstract color and environment color. The Hamilton Depression Scale (HAMD) and Hamilton Anxiety Scale (HAMA) were used to assess the symptoms and the patients were re-tested and re-assessed at six weeks after the antidepressant drug therapy. (See Table 1, Clinical Characteristics)

\subsubsection{Preference Test of Abstract Color}

The subjects' preference for abstract colors was investigated using the approach of paired comparison ${ }^{[5]}$. The color cards of red, orange, yellow, green, blue, purple, white, black and grey were selfmade. The nine kinds of color cards were put in pairs to make 36 combinations. They were divided into the photo albums in two groups, with 36 photos for each group. Each subject was required to complete 36 selections between Group A and Group B. The bench method was used to show the presentation sequence of arranged colors. Two colors of each card were marked as 1 and 0 , respectively. Supposing a subject chose the card 1 of Group A, if it indicated that the subject was more like the red, we marked it as 1 ; if it indicated that the subject was more like the orange, we marked it as 0 . Adding all scores of the same color together, the sum was the score of color preference. The higher the score was, the higher the preference degree would be.

\subsubsection{Preference Test of Indoor Environment Colors}

The digital images were used as the visual stimuli in the ranking method ${ }^{[6]}$ used to measure the color preference for each subject. Nine ward pictures of the same scene and different background wall colors were obtained, namely, red, orange, yellow, green, blue, purple, white, black and grey. A subject was asked to choose their favorite color from the nine pictures. All pictures were sorted or ranked according to the degree of preference, and they were marked as $1,2,3 \ldots$ until 9 in turn. Card 1 in the ranking sequence represented the most favorite color, and
Card 9 in the ranking sequence represented the least favorite color. Each of the nine colors in scores 1 - 9 was assigned. The lower the score was, the higher the degree of preference would be.

\subsubsection{Assessment of HAMD and HAMA}

The HAMD of 24 - item edition was used. Most items use level 5 (0 - 4 score ) ((0)without (1) light (2) moderate(3) severe (4) very serious). A few projects use level 3 (0 - 2 score ) (0) without, (1) light to moderate (2) severe. The severe depression is total score greater than 35 . The light to moderate depression is total score greater than 20 . The without depression is total score less than 8.The HAMA Scale included 14 items that reflected the anxiety symptoms. All items use level 5 (0 - 4 score ). The severe anxiety is total score greater than 29 . The obvious anxiety is total score greater than 21 . There must be some anxiety is total score greater than 14. There may be anxious is total score greater than 7 . The without anxiety is total score less than 7 .

\subsection{Statistical Analysis}

Software SPSS17.0 was used for the statistical analysis. Mean value \pm standard deviation $( \pm s)$ represents the color preference scores. The MannWhitney $U$ Test or t test was used for the comparison between groups; The Wilcoxon rank sum test was used for the comparison of preference sequence before and after the therapy; the Kendall rank correlation coefficient $(r)$ was used to analyze the relationship between color preference score and scale assessment score, and we used a significance level of $p<0.05$.

\section{RESULTS}

\subsection{Comparison of Abstract Color Preference Scores between Two Groups}

In the case group the preference for purple was significantly higher than that of control group ( $p=$ 0.023 ) and the preference of grey was significantly lower than that of control group $(p<0.01$. The difference of color preference of the patients before and after the therapy was not statistically significant $(p>0.05)$, see Table 2 . 
Table 2. Comparison of Abstract Color Preference Scores between Two Groups ( $\pm \mathbf{s})$

\begin{tabular}{lccccc}
\hline & \multicolumn{2}{c}{ Major Depression Group } & & \\
& Before Therapy & After Therapy & & & Control Group \\
Red & $4.60 \pm 1.98$ & $4.62 \pm 1.86$ & $4.67 \pm 1.91$ & 0.304 & 0.386 \\
Orange & $4.46 \pm 1.67$ & $4.39 \pm 1.69$ & $4.54 \pm 1.72$ & 0.497 & 0.513 \\
Yellow & $4.51 \pm 1.81$ & $4.47 \pm 1.71$ & $4.60 \pm 1.91$ & 0.311 & 0.744 \\
Green & $4.65 \pm 1.75$ & $4.65 \pm 1.58$ & $4.62 \pm 1.69$ & 0.080 & 0.435 \\
Blue & $4.74 \pm 1.68$ & $4.71 \pm 1.53$ & $4.67 \pm 1.76$ & 0.531 & 0.458 \\
Purple & $4.03 \pm 1.79$ & $3.96 \pm 1.82$ & $3.72 \pm 1.73$ & 0.207 & $2.279^{*}$ \\
White & $3.39 \pm 2.01$ & $3.45 \pm 1.97$ & $3.62 \pm 1.94$ & 0.529 & 1.688 \\
Black & $2.77 \pm 1.89$ & $2.89 \pm 1.90$ & $2.66 \pm 2.09$ & 0.911 & 1.017 \\
Grey & $2.85 \pm 1.83$ & $2.86 \pm 1.76$ & $3.80 \pm 1.88$ & 0.050 & $6.289^{* *}$ \\
\hline
\end{tabular}

Notes: Z1: comparison of Major depression group before and after therapy; Z2: Comparison with control group before therapy. ${ }^{*} p<0.05,{ }^{* *} p<0.01$.

\subsection{Comparison of Abstract Color Preference Sequence between Two Groups}

The abstract color preference sequence of study group was sorted from high to low: blue, green, red, yellow, orange, purple, white, grey and black. Compared with the control group, the rank correlation coefficient $r=0.833$, and statistically significant test $u=3.023>2.58$, so $p<0.01$, which indicated that the rank correlation of color preference sequence between Major depression group and control group was statistically significant and their tendencies in color choice were consistent. The color preference sequence after therapy was sorted from high to low: blue, green, red, yellow, orange, purple, white, black and grey. Compared with the preference sequence before therapy, the rank correlation coefficient $r=0.944$ after rank correlation treatment and statistically significant test $u=3.440>2.58$, so $p<0.01$, which indicated that preference sequence tendencies before and after therapy were consistent and they both preferred for blue.

\subsection{Comparison of Indoor Environment Color Preference between Two Groups}

The preference of Major depression group for the purple was significantly higher than that of control group ( $p=0.014)$ and the preference of Major depression group for the orange was significantly lower than that of control group $(p=0.005)$. The preference for the red and grey after therapy decreased compared with the preference before therapy $(p=0.003,0.040)$ and the preference for the yellow increased compared with the preference before therapy $(p=0.002)$, see Table 3 . 
Table 3. Comparison of Indoor Environment Color Preference Scores between Two Groups ( \pm s)

\begin{tabular}{|c|c|c|c|c|c|}
\hline & \multicolumn{2}{|c|}{ Major Depression Group } & \multirow{2}{*}{ Control Group } & \multirow{2}{*}{$\mathrm{Z1}$} & \multirow{2}{*}{$\mathrm{Z2}$} \\
\hline & Before Therapy & After Therapy & & & \\
\hline Red & $5.03 \pm 2.32$ & $5.41 \pm 2.13$ & $5.10 \pm 2.30$ & $2.934^{*}$ & 0.468 \\
\hline Orange & $4.93 \pm 2.00$ & $4.69 \pm 2.04$ & $4.46 \pm 2.11$ & 1.584 & $2.835^{* *}$ \\
\hline Yellow & $4.62 \pm 2.07$ & $4.10 \pm 1.78$ & $4.51 \pm 2.16$ & $3.055^{*}$ & 0.747 \\
\hline Green & $4.14 \pm 2.08$ & $4.11 \pm 2.01$ & $4.38 \pm 2.23$ & 0.381 & 1.291 \\
\hline Blue & $3.97 \pm 2.34$ & $3.79 \pm 2.19$ & $4.15 \pm 2.33$ & 0.937 & 1.018 \\
\hline Purple & $4.22 \pm 2.51$ & $4.32 \pm 2.49$ & $4.69 \pm 2.49$ & 0.753 & $2.457^{* *}$ \\
\hline White & $4.82 \pm 2.81$ & $4.66 \pm 2.89$ & $4.42 \pm 2.75$ & 0.763 & 1.836 \\
\hline Black & $6.63 \pm 2.91$ & $6.81 \pm 2.97$ & $6.62 \pm 2.81$ & 0.899 & 0.098 \\
\hline Grey & $6.72 \pm 2.46$ & $7.08 \pm 2.14$ & $6.70 \pm 2.44$ & $2.059^{*}$ & 0.264 \\
\hline
\end{tabular}

Notes: Z1: comparison of Major depression group before and after therapy; Z2: Comparison with control group before therapy. ${ }^{*} p<0.05,{ }^{* *} p<0.01$.

\subsection{Comparison of Indoor Environment Color Preference Sequence between Two Groups}

The indoor environment color preference sequence of Major depression group was sorted from high to low: blue, green, purple, yellow, white, orange, red, black and grey. Compared with the control group, the rank correlation coefficient $r=0.722$, and statistically significant test $u=2.606>2.58$, so $p<0.01$, which indicated that the rank correlation of color preference sequence between Major depression group and control group was statistically significant and their tendencies in color choice were consistent.

The color preference sequence after therapy was sorted from high to low: blue, yellow, green, purple, white, orange, red, black and grey. Compared with the preference sequence before therapy, the rank correlation coefficient $r=0.861$ and statistically significant test $u=3.128>2.58$, so $p<0.01$, which indicated that color preference sequence tendencies before and after therapy were consistent and they both preferred for blue.

\subsection{Analysis to Correlation between Patients' Color Preference Scores and HAMD and HAMA Scores}

1) Scale scores before and after therapy: the difference of Major depression group for HAMD scores before and after therapy was statistically significant $[(25.42 \pm 8.28)$ VS $(10.18 \pm 9.19), t=$ 23.272, $p<0.001$ ]; and the difference of Major depression group for HAMA scores before and after therapy was statistically significant $[(16.95 \pm 7.38)$ VS $(6.68 \pm 6.55), t=18.539, p<0.001]$, see Table 4 .

Table 4.HAMD and HAMA Scores before and after therapy

\begin{tabular}{lcccc}
\hline & Before Theray & After Theray & $\mathrm{t}$ & $p$ \\
\hline HAMD score & $25.42 \pm 8.28$ & $10.18 \pm 9.19$ & 23.272 & $p<0.001$ \\
HAMA score & $16.95 \pm 7.38$ & $6.68 \pm 6.55^{*}$ & 18.539 & $p<0.001$ \\
\hline
\end{tabular}

Notes: Comparison of Major depression group before and after therapy: ${ }^{*} p<0.01$. 
A significantly negative correlation was found between yellow preference sequence and HAMD score and between yellow preference sequence and HAMA score $(p=0.047,0.014)$. A significantly positive correlation was found between red preference sequence and HAMD score $(p=0.000)$, and a significantly negative correlation was found between blue/white preference sequence and HAMD score $(p=0.012,0.023)$, see Table 5 .

Table 5. Correlation ( $r$ ) between Patients' Color Preference Scores and HAMD and HAMA Scores

\begin{tabular}{llllllllllll}
\hline Experiment & $\begin{array}{l}\text { Impact } \\
\text { Factor }\end{array}$ & Red & Orange & Yellow & Green & Blue & Purple & White & Black & Grey \\
\hline $\begin{array}{l}\text { Abstract color } \\
\text { preference }\end{array}$ & $\begin{array}{l}\text { HAMD } \\
\text { score }\end{array}$ & 0.054 & -0.060 & $-0.133^{*}$ & 0.064 & 0.037 & 0.095 & -0.035 & 0.076 & -0.104 \\
& $\begin{array}{l}\text { HAMA } \\
\text { score }\end{array}$ & 0.100 & 0.081 & $-0.182^{*}$ & -0.027 & -0.046 & 0.090 & 0.022 & 0.044 & -0.091 \\
& $\begin{array}{l}\text { HAMD } \\
\text { Environment } \\
\text { color } \\
\text { preference }\end{array}$ & $0.248^{* *}$ & 0.056 & 0.085 & 0.003 & $-0.167^{*}$ & -0.096 & $-0.152^{*}$ & 0.073 & 0.044 \\
& $\begin{array}{l}\text { score } \\
\text { SAMA }\end{array}$ & 0.108 & -0.033 & 0.028 & 0.008 & -0.069 & 0.019 & -0.137 & 0.079 & 0.048 \\
\hline
\end{tabular}

Notes: ${ }^{*} p<0.05,{ }^{* *} p<0.01$.

\section{DISCUSSION}

This study shows that the preference sequence and preference tendency of depression patients for the abstract color are consistent, and there is no significant overall difference from the color preferences of healthy people. However, there are differences in preferences for some colors (purple, grey). The depression patients prefer blue the most, and their preference for purple is higher than that of the healthy people, blue is often of a symbolic significance of melancholy and pessimism, and the symbolic significance of purple is anxiety and nervousness ${ }^{[7]}$. The mood-congruent theory states that depression individuals can recall or pay attention to the negative stimulus consistent with their depressed mood, and it is manifested as a more stable phenomenon and it is regarded as an important cognitive mechanism that maintains the state of depression ${ }^{[8]}$. The depression patients were significantly different before and after therapy, but their preference sequence for abstract colors did not change significantly. Except for position exchange of grey and black, the sequencing of other colors did not change. It is suggested that the preference of depression patients for abstract colors may not be significantly associated with their psychological and emotional status.
The test results of indoor environment color preference showed that the blue was again the most preferred color of the patients with depressive disorders and the grey was their least preferred color. Only the ranking of yellow changed six weeks after therapy, where the preference of red and grey decreased significantly and the preference for yellow increased significantly. The negative psychological effect of grey is depression and pessimism the red can accelerate the heartbeats and raise the blood pressure, which gives people the feeling of excitement, enthusiasm or anger. An excessive exposure to the red will cause anxiety and physical I mental stress, causing an individual to be tired out. Hence, the color preference of patients for red and grey decreased after the remission of emotional symptoms. The preference of depression patients for indoor environment color was closely associated with their emotional and psychological states. Moreover, the preference of depression patients for the purple was significantly higher than that of healthy volunteers, while their preference for the orange was significantly lower than that of control group.

This study further showed that whether it was abstract color or indoor environment color, the patients with depressive disorders preferred blue, and their preference for purple was significantly 
higher than that of healthy people. It was indicated the depression patients preferred the cold colors. This might be due to the psychological belief that the cold colors could stabilize and calm down a restless or uneasy mood ${ }^{[9]}$. However, some studies also pointed out that the patients with depressive disorders should not be exposed in blue or other cold colors, otherwise it could aggravate the disease conditions $^{[10]}$. The study results of Nolan, et al. pointed out that yellow was soft warm color that could reduce the anxiety level of patients ${ }^{[1]}$. The analysis of correlation between color preference scores and symptom scores of depression patients shows that in the aspect of abstract color, a significantly negative correlation was found between yellow preference and HAMD score and between yellow preference and HAMA score. This indicates that the preference test of abstract yellow may have a certain predictive value for the assessment of depression mood. In the aspect of environment color, a significantly positive correlation was found between red preference sequence and HAMD score, while the blue and white preference sequences were significantly negative correlated. This indicates that, the higher the depression score is, the more unfavorable to the red, and the more favorable to the blue, white and other cold colors. This was consistent with the study result of Dai Hong, et al.

[11] Therefore, it is recommended that the application of colors to the ward environment design of patients with depressive disorders can play a role of adjuvant therapy in anxiety and depression of patients with depressive disorders.

There have a little deficiencies in this study. The antidepressant may have an effect on color preference, so the experiment needs to be further improved and confirmed. In addition, further research is needed to clarify the correlation between color application and depression emotional state, and to explore the value and potential of application color for psychotherapy in patients with depression.

\section{FUNDING SUPPORT}

This study was supported by government (Key research project of Chongqing health and Family Planning Commission) (Program No. 2013-1-045).

\section{REFERENCES}

1. Nolan RF, Dai Y, Stanley PD. An investigation of the relationship between color choice and depression measured by the Beck Depression Inventory. Percept Mot Skills. 1995; 81(3f): 11951200.

2. Carruthers HR, Morris J, Tarrier N, Whorwell PJ. The Manchester Color Wheel: development of a novel way of identifying color choice and its validation in healthy, anxious and depressed individuals. BMC Med Res Methodol. 2010; 10(1): 12.

3. Von Goethe JW. Theory of Colours. Cambridge, MA: MIT Press. 1970: 1840.

4. Taylor JL, John CH. Attentional and memory bias in persecutory delusions and depression. Psychopathol. 2004; 37(5): 233-241.

5. Alarcón M, Abrahams BS, Stone JL, Duvall JA, Perederiy JV, Bomar JM, Sebat J, Wigler M, Martin CL, Ledbetter DH, Nelson SF, Cantor $\mathrm{RM}$, Geschwind DH. Linkage, association, and gene2 expression analyses identify CNTNAP2 as an autism 2 susceptibility gene. A m J Hum Genet. 2008; 82: 150-159.

6. Xu AB. Color and Personality - The Applied Research of Artificial Neural Networks in Psychological Test. Technol Inf. 2011; 35: 328330.

7. Yan WN. On the Color Psychology in Life. J Tonghua Normal University. 2008; 6: 52-54.

8. He ZH, Zhang DD, Luo YJ. Mood-Congruent Cognitive Bias in Depressed Individuals. Adv Psychol Sci. 2015; 23(12): 2118-2128.

9. Nagumo H (Japan). Training and Teaching Courses of Color Design of Colleges and Universities in Japan. Shanghai People's Fine Arts Publishing House, 2006.

10. Zhou N. On the Relationship between Color and Physical \& Mental Health. Art Edu Res. 2013; (22): 33-33.

11. Dai H, Xu SF. Color Preferences and Their Prediction of Emotions in Patients with Major Depressive and Generalized Anxiety Disorders. J Sun Yat-Sen Univ: Med Sci. 2015; 36(3): 427431.

\section{CONFLICT OF INTERESTS}

Authors claim no conflict of interests. 